\title{
Soret and Chemical Reaction Effects on Unsteady MHD Flow of Viscoelastic Micropolar Fluid through a Porous Medium with Thermal Radiation and Heat Source.
}

\author{
D.Babu Reddy and G.S.S.Raju \\ Department of Mathematics,JNTUA College of Engineering,Pulivendula,YSR Kadapa Dist, A.P., ,INDIA
}

\begin{abstract}
The objective of the present paper is to analyze the heat and mass transfer effects on an unsteady MHD flow of a chemically reacting micropolar fluid past an infinite vertical porous plate through a porous media in the presence of thermal radiation with Hall current, viscoelastic and Soret effects by taking into account. The governing equations are transformed into dimensionless equations and then solved analytically using Perturbation technique. The expressions for the velocity, microrotation, temperature and concentration have been derived and its behavior is computationally discussed with reference to different pertinent flow parameters with the help of graphs. Also the influence of the various flow parameters on the skin friction coefficient, couples stress coefficient, Nusselt number, and Sherwood number are presented numerically in a tabular form.
\end{abstract}

Keywords: Micropolar fluid, Perturbation technique, Hall current and Soret effects, Chemical reaction, Porous medium, heat source

\section{Introduction}

The micropolar fluid theory is the one of the most important non-Newtonian fluid models describe by Eringen [1].This theory shows microrotation effects as well as micro inertia and has many applications such as polymer fluids, liquid crystals, animal bloods, unusual lubricants, colloidal and suspension solutions, colloidal fluids, liquid crystals, and polymeric suspension. The extensive reviews of the micropolar fluid theory and its applications can be found in Eringen [2].The comprehensive literature on micropolar fluids, thermomicropolar fluids and their applications in engineering and technology were presented by [3, 4]. Srinivasacharya et al. [5] analyzed the unsteady stokes flow of micropolar fluid between two parallel porous plates. Muthuraj and Srinivas [6] investigated fully developed MHD flow of a micropolar and viscous fluid in a vertical porous space using HAM.

Heat and mass transfer from different geometries embedded in porous media has many engineering and geophysical applications such as drying of porous solids, thermal insulations, cooling of nuclear reactors, crude oil extraction, underground energy transport, etc .Several authors have studied the characteristic of the boundary layer flow of heat and mass transfer micropolar fluid under different boundary conditions [7-12]

The combined heat and mass transfer problems with chemical reaction are of importance in many processes and have, therefore, received a considerable amount of attention in recent years. In processes, such as drying, evaporation at the surface of a water body, energy transfer in a wet cooling the tower, and the flow in a desert cooler, the heat and mass transfer occurs simultaneously. Damesh et al. [13] have studied the combined effect of heat generation or absorption and first order chemical reaction to micropolar fluid flows over a uniform stretched surface. Some other related works can also be found in the papers [14-16].

In all the previous investigations, the effect of thermal radiation on the flow and heat transfer has not been provided. The effect of radiation on MHD flow and heat transfer problem has become more important industrially. At high operating temperatures, radiation effect can be quite significant, many processes in engineering areas occur at high temperatures and knowledge of radiation heat transfer become very important for design of reliable equipment, nuclear plants, gas turbines and various propulsion devices or aircrafts, missiles, satellites, and space vehicles. Abo-eldohad and Ghonaim [17] analyzed the radiation effects on heat transfer of a micropolar fluid through a porous medium. Rahman and Sultana [18] have studied the steady convective flow of a micropolar fluid past a vertical porous flat plate in the presence of radiation with variable heat flux in porous medium. The effects of thermal radiation were also investigated by the researchers $[19,20]$.

When the strength of the magnetic field is strong, one cannot neglect the effect of Hall current. It is of considerable importance and interest to study how the results of the hydro- dynamical problems get modified by the effect of Hall currents. Hall currents give rise to a cross flow making the flow three dimensional. Several authors [21-23] studied MHD flow of a micropolar fluid. Rakesh [24] studied effect of slip conditions and Hall current on unsteady MHD flow of a viscoelastic fluid past an infinite vertical porous plate through porous medium. The effects of Hall current, rotation and radiation absorption on MHD micropolar fluid past a vertical 
porous plate was studied by P.V. Satya Narayana et al [25]. B.I.Olajuwon et al [26] extended the work of Rakesh [24] by incorporate angular momentum and concentration equations with thermal radiation term to study Hall current and thermal radiation effect on heat and mass transfer of unsteady MHD flow of a viscoelastic micropolar fluid through a porous medium.

The aim of the present analysis is to investigate Soret and chemical reaction effects on unsteady MHD viscoelastic micropolar fluid through a porous medium. We have extended the work of B.I.Olajuwon et al [26] with the novelty of considering Soret effect along with chemical reaction in presence of heat Source, which appears to be used in many areas of science and technology. As far as author's knowledge is concern this phenomenon was not studied earlier, hence we showed attention and attempted to study this work.

\section{Formulation Of The Problem}

We consider an unsteady hydromagnetic flow of a viscous, incompressible, and electrically conducting viscoelastic micropolar fluid past an infinite vertical porous plate, subjected to a uniform transverse magnetic field $\mathrm{B}_{0}$ taking Hall current, thermal radiation, Heat source, Soret effect and chemical reaction taking into account. The induced magnetic field is assumed to be negligible compared to the applied magnetic field. We choose a coordinate system in such a way that $\bar{x}$-axis is considered along the plate in vertically upward direction, and the $\bar{y}$-axis is taken along the width of the plate, $\bar{z}$-axis normal to the plane of the plate in the fluid as shown in Fig. 1. Due to the infinite plane surface assumption, the flow variables are function of $\bar{z}$ and $\bar{t}$ only. The plate is subjected to a constant suction velocity $w_{0}$.

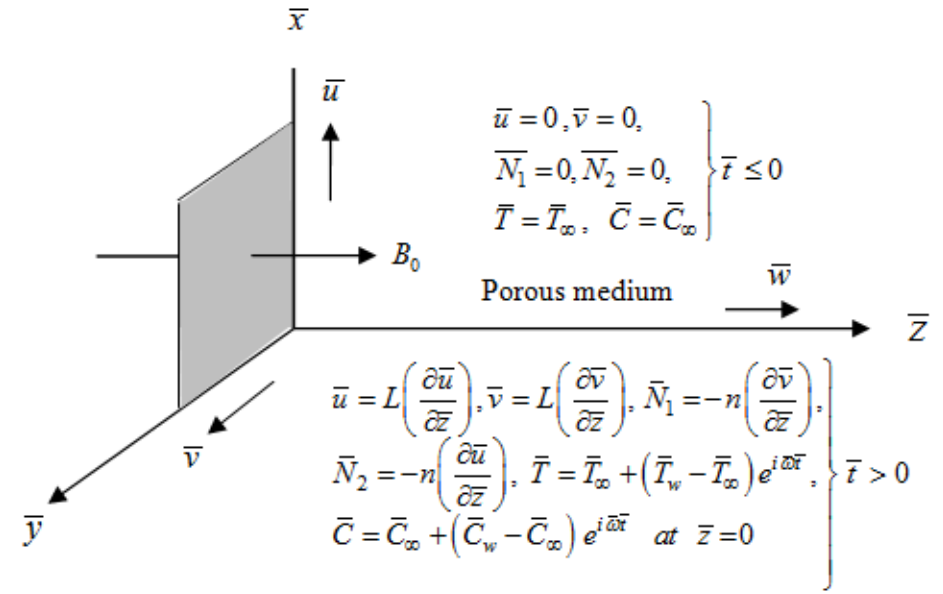

FIG. 1: Physical model of the problem

A magnetic field of constant strength $\mathrm{B}_{0}$ is applied parallel to $\bar{z}$-axis which is perpendicular to the flow direction. It is assumed that the Induced magnetic field due to the flow is neglected compared to the applied magnetic field. This assumption is physically justified for liquid metals and partially ionized fluids which are commonly used in industrial applications, because their magnetic Reynolds number is very small. With these foregoing assumptions, the governing equations of flow under the usual Boussinesq approximation can be written in a Cartesian frame of reference are given by

$$
\begin{aligned}
& \frac{\partial \bar{w}}{\partial \bar{z}}=0 \\
& \frac{\partial \bar{u}}{\partial \bar{t}}+\bar{w} \frac{\partial \bar{u}}{\partial \bar{z}}=\left(v+v_{r}\right) \frac{\partial^{2} \bar{u}}{\partial \bar{z}^{2}}-K_{0} \frac{\partial^{3} \bar{u}}{\partial \bar{t} \partial \bar{z}^{2}}+v_{r} \frac{\partial \overline{N_{2}}}{\partial \bar{z}} \\
& +g \beta\left(\bar{T}-\bar{T}_{\infty}\right)+g \bar{\beta}\left(\bar{C}-\bar{C}_{\infty}\right)+\frac{\sigma B_{0}^{2}}{\rho\left(1+m^{2}\right)}(m \bar{v}-\bar{u})-\frac{v \bar{u}}{\bar{K} r} \\
& \frac{\partial \bar{v}}{\partial \bar{t}}+\bar{w} \frac{\partial \bar{v}}{\partial \bar{z}}=\left(v+v_{r}\right) \frac{\partial^{2} \bar{v}}{\partial \bar{z}^{2}}-K_{0} \frac{\partial^{3} \bar{v}}{\partial \bar{t} \partial \bar{z}^{2}}+v_{r} \frac{\partial \overline{N_{1}}}{\partial \bar{z}}-\frac{\sigma B_{0}^{2}}{\rho\left(1+m^{2}\right)}(m \bar{u}+\bar{v})-\frac{v \bar{v}}{\bar{K} r} \\
& \frac{\partial \bar{N}_{1}}{\partial \bar{t}}+\bar{w} \frac{\partial \bar{N}_{2}}{\partial \bar{z}}=\frac{\gamma}{\rho \bar{J}} \frac{\partial^{2} \bar{N}_{1}}{\partial \bar{z}^{2}} \\
& \frac{\partial \bar{N}_{2}}{\partial \bar{t}}+\bar{w} \frac{\partial \bar{N}_{2}}{\partial \bar{z}}=\frac{\gamma}{\rho \bar{J}} \frac{\partial^{2} \bar{N}_{2}}{\partial \bar{z}^{2}}
\end{aligned}
$$




$$
\begin{aligned}
& \frac{\partial \bar{T}}{\partial \bar{t}}+\bar{w} \frac{\partial \bar{T}}{\partial \bar{z}}=\frac{k}{\rho C p} \frac{\partial^{2} \bar{T}}{\partial \bar{z}^{2}}-\frac{1}{\rho C p} \frac{\partial q_{r}}{\partial \bar{z}}+\frac{\bar{Q}}{\rho C p}\left(\bar{T}-\bar{T}_{\infty}\right) \\
& \frac{\partial \bar{C}}{\partial \bar{t}}+\bar{w} \frac{\partial \bar{C}}{\partial \bar{z}}=D_{m} \frac{\partial^{2} \bar{C}}{\partial \bar{z}^{2}}+D_{T} \frac{\partial^{2} \bar{T}}{\partial \bar{z}^{2}}-\bar{C}_{r}\left(\bar{C}-\bar{C}_{\infty}\right)
\end{aligned}
$$

The initial and boundary conditions are:

$$
\left.\begin{array}{rrr}
\bar{t} \leq 0: \bar{u}=0, \bar{v}=0, \overline{N_{1}}=0, \overline{N_{2}}=0, \bar{T}=\bar{T}_{\infty}, \bar{C}=\bar{C}_{\infty} & \text { for all } \bar{z} \\
\bar{t}>0: \bar{u}=\bar{L}\left(\frac{\partial \bar{u}}{\partial z}\right), \bar{v}=\bar{L}\left(\frac{\partial \bar{v}}{\partial \bar{z}}\right), \overline{N_{1}}=-n\left(\frac{\partial \bar{v}}{\partial z}\right), \overline{N_{2}}=-n\left(\frac{\partial \bar{u}}{\partial \bar{z}}\right), & \\
\bar{T}=\bar{T}_{\infty}+\left(\bar{T}_{w}-\bar{T}_{\infty}\right) e^{i \bar{\omega} \bar{t}}, \bar{C}=\bar{C}_{\infty}+\left(\bar{C}_{w}-\bar{C}_{\infty}\right) e^{i \bar{\omega} \bar{t}} & \text { at } \bar{z}=0 \\
\bar{t}>0: \bar{u} \rightarrow 0, \bar{v} \rightarrow 0, \bar{N}_{1} \rightarrow 0, \overline{N_{2}} \rightarrow 0, \bar{T} \rightarrow \bar{T}_{\infty}, \bar{C} \rightarrow \bar{C}_{\infty} & \text { as } \bar{z} \rightarrow \infty
\end{array}\right\}
$$

where $\bar{u}, \bar{v}$ and $\bar{w}$ denote the velocity components of the fluid in the $\bar{x}, \bar{y}$ and $\bar{z}$ directions respectively, $\bar{N}_{1}$ and $\bar{N}_{2}$ are micro rotation components along $\bar{x}$ and $\bar{y}$ respectively, $v$ is the kinematic viscosity, $v_{r}$ is the kinematic micro-rotation viscosity, $K_{0}$ is the limiting viscosity, $g$ is the acceleration due to gravity, $\beta$ is the volumetric coefficient of thermal expansion, $\bar{\beta}$ is the volumetric coefficient of concentration expansion, $\sigma$ is the electrical conductivity, $m=\omega_{e} \tau_{e}$ is the Hall parameter, $\omega_{e}$ is the cyclotron frequency, $\tau_{e}$ is the electron collision time, $\bar{K}_{r}$ is the permeability of the porous medium, $\bar{T}$ is the dimensional temperature of the fluid, $\bar{T}_{w}$ and $\bar{T}_{\infty}$ denotes the temperature at the plate and temperature far away from the plate respectively, $k$ is the thermal conductivity, $\rho$ is the fluid density, $\bar{C}$ is the dimensional concentration of the solute, $\bar{C}_{w}$ and $\bar{C}_{\infty}$ are concentration of the solute at the plate and concentration of the solute far from the plate respectively, $\bar{J}$ is the micro-inertia density, $\gamma$ is the spin gradient viscosity, $\bar{L}$ is the characteristic length, $\bar{\omega}$ is the dimensional frequency of oscillation, $C_{p}$ is specific heat at constant pressure, $q_{r}$ is the radiative heat flux , $D_{M}$ is the coefficient of mass diffusivity, $D_{T}$ is the thermal diffusion ratio and $\bar{C}_{r}$ is the chemical reaction parameter.

The constant that related to micro gyration vector and shear stress is $n$, where $0 \leq n \leq 1$. The case $n=0$ represents concentrated particle flows in which the microelement close to the wall surface are unable to rotate. This case is also known as the strong concentration of microelements. The case $\mathrm{n}=0.5$ indicates the vanishing of anti-symmetric part of the stress tensor and denotes weak concentration of microelements. The case $n=1$ is used for the modeling of turbulent boundary layer flows. We shall consider $\mathrm{n}=0$ and $\mathrm{n}=0.5$.

Integrating of continuity eq. (1) for constant suction velocity normal to the plate gives, we obtain

$$
\bar{w}=-w_{0}
$$

where $w_{0}$ represents the velocity normal to the plate which is positive for suction and negative for blowing. The radiative heat flux term by using Rosseland approximation is given by

$$
q_{r}=-\frac{4 \sigma_{1}}{k_{1}}\left(\frac{\partial \bar{T}^{4}}{\partial \bar{z}}\right)
$$

Where $\sigma_{1}$ is Stefan-Boltzmann constant and $k_{1}$ mean absorption coefficient.The temperature difference within the flow is assumed to be sufficiently small, then equation (6) can be linearized by expanding $\bar{T}^{4}$ into the Taylor series about $\overline{T_{\infty}}$, which after neglecting the higher order terms, thus

$$
\bar{T}^{4}=-3 \bar{T}_{\infty}^{4}+4 \bar{T} \bar{T}_{\infty}^{3}
$$

Differentiating equation (10) with respect to $\bar{z}$ and using eq.(11) to obtain

$$
\frac{\partial q_{r}}{\partial z}=-\frac{16 \bar{T}_{\infty}^{3} \sigma_{1}}{3 k_{1}}\left(\frac{\partial^{2} \bar{T}}{\partial \bar{z}^{2}}\right)
$$

Proceeding with analysis, we introduce the following dimensionless variables:

$$
\begin{aligned}
& u=\frac{\bar{u}}{w_{0}}, v=\frac{\bar{v}}{w_{0}}, \mathrm{z}=\frac{w_{0} \bar{z}}{v}, t=\frac{\bar{t} w_{0}^{2}}{4 v}, N_{1}=\frac{v \overline{N_{1}}}{w_{0}^{2}}, N_{2}=\frac{v \overline{N_{2}}}{w_{0}^{2}}, \\
& \left.J=\frac{\bar{J} w_{0}^{2}}{v^{2}}, \omega=\frac{4 v \bar{\omega}}{w_{0}^{2}}, h=\frac{w_{0} \bar{L}}{v}, \theta=\frac{\bar{T}-\bar{T}_{\infty}}{\bar{T}_{w}-\bar{T}_{\infty}}, \phi=\frac{\bar{C}-\bar{C}_{\infty}}{\bar{C}_{w}-\bar{C}_{\infty}}\right\}
\end{aligned}
$$


In view of eq. (13), the governing equations (2) to (7) yields to the following dimensionless equations:

$$
\begin{aligned}
& \frac{1}{4} \frac{\partial u}{\partial t}-\frac{\partial u}{\partial z}=(1+\beta) \frac{\partial^{2} u}{\partial z^{2}}-a \frac{\partial^{3} u}{\partial t \partial z^{2}}-\beta \frac{\partial N_{2}}{\partial z}+G r \theta+G m \phi+\frac{M}{\left(1+m^{2}\right)}(m v-u)-\frac{u}{K r} \\
& \frac{1}{4} \frac{\partial v}{\partial t}-\frac{\partial v}{\partial z}=(1+\beta) \frac{\partial^{2} v}{\partial z^{2}}-a \frac{\partial^{3} v}{\partial t \partial z^{2}}+\beta \frac{\partial N_{1}}{\partial z}-\frac{M}{\left(1+m^{2}\right)}(m u+v)-\frac{v}{K r} \\
& \frac{1}{4} \frac{\partial N_{1}}{\partial t}-\frac{\partial N_{1}}{\partial z}=L \frac{\partial^{2} N_{1}}{\partial z^{2}} \\
& \frac{1}{4} \frac{\partial N_{2}}{\partial t}-\frac{\partial N_{2}}{\partial z}=L \frac{\partial^{2} N_{2}}{\partial z^{2}} \\
& \frac{1}{4} \frac{\partial \theta}{\partial t}-\frac{\partial \theta}{\partial z}=\left(\frac{1+R}{\operatorname{Pr}}\right) \frac{\partial^{2} \theta}{\partial z^{2}}+\frac{Q}{\operatorname{Pr}} \theta \\
& \frac{1}{4} \frac{\partial \phi}{\partial t}-\frac{\partial \phi}{\partial z}=\frac{1}{S c} \frac{\partial^{2} \phi}{\partial z^{2}}+S r \frac{\partial^{2} \theta}{\partial z^{2}}-C r \phi
\end{aligned}
$$

where $\beta=v_{r} / v$ is the dimensionless viscosity ratio, $a=K_{0} w_{0}{ }^{2} / 4 v^{2}$ is the viscoelastic parameter, $M=\sigma B_{0}^{2} v / \rho w_{0}^{2}$ is the magnetic field parameter, $K r=\overline{K r} w_{0}^{2} / v^{2}$ is the permeability of the porous medium, $G r=g \beta v\left(\bar{T}_{w}-\bar{T}_{\infty}\right) / w_{0}^{3}$ is the Grashof number, $G m=g \bar{\beta} v\left(\bar{C}_{w}-\bar{C}_{\infty}\right) / w_{0}^{3}$ is the modified Grashof number, $L=\gamma w_{0}^{2} / \rho v^{3} J$ is the material parameter, $\operatorname{Pr}=v \rho C p / k$ is the Prandtl number, $R=16 \bar{\sigma} \bar{T}_{\infty}^{3} / 3 k k_{1}$ is thermal radiation parameter, $Q=\bar{Q} v^{2} / k w_{0}^{2}$ is heat source parameter, $S c=v / D_{M}$ is the Schmidt number, $\operatorname{Sr}=D_{T}\left(\bar{T}_{w}-\bar{T}_{\infty}\right) / v\left(\bar{C}_{w}-\bar{C}_{\infty}\right)$ is the Soret number, $C r=\bar{C}_{r} v / w_{0}^{2}$ is the chemical reaction parameter and $h=w_{0} \bar{L} / v$ is the slip parameter.

The boundary conditions (8)-(9) in view of (14) are then given by the following dimensionless form:

$$
\begin{aligned}
& t \leq 0: u=0, v=0, N_{1}=0, N_{2}=0, T=T_{\infty}, C=C_{\infty} \\
& \left.\begin{array}{rlr}
t>0: & =h\left(\frac{\partial u}{\partial z}\right), v=h\left(\frac{\partial v}{\partial z}\right), N_{1}=-n\left(\frac{\partial v}{\partial z}\right), N_{2}=n\left(\frac{\partial u}{\partial z}\right), \theta=e^{i \omega t}, \phi=e^{i \omega t} & \text { at } z=0 \\
: & \rightarrow 0, v \rightarrow 0, N_{1} \rightarrow 0, N_{2} \rightarrow 0, \theta \rightarrow 0, \phi \rightarrow 0 \quad \text { as } \quad z \rightarrow \infty
\end{array}\right\}
\end{aligned}
$$

To simplify eq. (14) - eq. (17), we substitute the fluid velocity and angular velocity in the complex form as $F=u+i v$ and $P=N_{1}+N_{2}$ we get

$$
\begin{aligned}
& \frac{1}{4} \frac{\partial F}{\partial t}-\frac{\partial F}{\partial z}=(1+\beta) \frac{\partial^{2} F}{\partial z^{2}}-a \frac{\partial^{3} F}{\partial t \partial z^{2}}+G r \theta+G m \phi-\left(\frac{M^{2}(1-i m)}{1+m^{2}}+\frac{1}{K r}\right) F+i \beta \frac{\partial P}{\partial z} \\
& \frac{1}{4} \frac{\partial P}{\partial t}-\frac{\partial P}{\partial z}=L \frac{\partial^{2} P}{\partial z^{2}} \\
& \frac{1}{4} \frac{\partial \theta}{\partial t}-\frac{\partial \theta}{\partial z}=\left(\frac{1+R}{\operatorname{Pr}}\right) \frac{\partial^{2} \theta}{\partial z^{2}}+\frac{Q}{\operatorname{Pr}} \theta \\
& \frac{1}{4} \frac{\partial \phi}{\partial t}-\frac{\partial \phi}{\partial z}=\frac{1}{S c} \frac{\partial^{2} \phi}{\partial z^{2}}+S r \frac{\partial^{2} \theta}{\partial z^{2}}-C r \phi
\end{aligned}
$$

The initial and boundary conditions (14), in compact form becomes,

$$
\left.\begin{array}{c}
t \leq 0: F=0, P=0, \theta=0, \phi=0 \quad \text { for all } z \\
t>0: F=h \frac{\partial F}{\partial z}, P=i n \frac{\partial P}{\partial z}, \theta=e^{i \omega t}, \phi=e^{i \omega t} \quad \text { at } z=0 \\
: F \rightarrow 0, P \rightarrow 0, \theta \rightarrow 0, \phi \rightarrow 0 \quad \text { as } z \rightarrow \infty
\end{array}\right\}
$$$$
\text { III. Method Of Solution }
$$

In order to reduce the above system of partial differential equations to a system of ordinary differential equations in dimensionless form, we assume a perturbation method of this form:

$$
F=F_{0}(z) e^{i \omega t}, P=P_{0}(z) e^{i \omega t}, \theta=\theta_{0}(z) e^{i \omega t}, \phi=\phi_{0}(z) e^{i \omega t}
$$


Substituting the above equations into the Eqs.(21),(22),(23) and (24),we obtain the following set of equations :

$A_{1} F_{0}^{\prime \prime}+F_{0}^{\prime}-A_{2} F_{0}=-i \beta P_{0}^{\prime}-G r \theta_{0}-G c \phi_{0}$

$L P_{0}^{\prime \prime}+P_{0}^{\prime}-\frac{i \omega}{4} P_{0}=0$

$A_{3} \theta_{0}^{\prime \prime}+\theta_{0}^{\prime}+A_{4} \theta_{0}=0$

$\phi_{0}^{\prime \prime}+S c \phi_{0}^{\prime}-A_{5} \phi_{0}=A_{6} \theta_{0}^{\prime \prime}$

Where $A_{1}=1+\beta-i a \omega, A_{2}=\left(M^{2}(1-i m) / 1+m^{2}\right)+1 / K r+i \omega / 4, A_{3}=(1+R) / \operatorname{Pr}$,

$$
A_{4}=Q-i \omega / 4, A_{5}=C r S c+i \omega S c / 4, A_{6}=-S r S c .
$$

The corresponding boundary conditions can be written as

$$
\left.\begin{array}{l}
F_{0}=h \frac{\partial F_{0}}{\partial z}, P_{0}=i n \frac{\partial P_{0}}{\partial z}, \theta_{0}=1, \phi_{0}=1 \quad \text { at } \quad z=0 \\
F_{0} \rightarrow 0, P_{0} \rightarrow 0, \theta_{0} \rightarrow 0, \phi_{0} \rightarrow 0 \quad \text { as } z \rightarrow \infty
\end{array}\right\}
$$

The solution of satisfying the boundary conditions eq.(25) are given by

$$
\begin{aligned}
F & =\left[m_{1} \exp \left(-r_{4} z\right)+m_{2} \exp \left(-r_{2} z\right)+m_{3} \exp \left(-r_{3} z\right)+m_{4} \exp \left(-r_{1} z\right)\right] \exp (\mathrm{i} \omega t) \\
P & =B \exp \left(i \omega t-r_{1} z\right) \\
\theta & =\exp \left(i \omega t-r_{2} z\right) \\
\phi & =a_{1} \exp \left(i \omega t-r_{2} z\right)+a_{2} \exp \left(i \omega t-r_{3} z\right)
\end{aligned}
$$

The Physical quantities of practical interest, in this study, are skin friction coefficient, couple stress coefficient, Nusselt Number and Sherwood number which are calculated as:

The skin friction coefficient $(\mathrm{Cf})$ at the plate is given by

$$
C f=\frac{\tau_{w}}{\rho W_{0}^{2}}=(1+(1-n) L) q^{\prime}(0)=-(1+(1-n) L)\left(r_{4} m_{1}+r_{2} m_{2}+r_{3} m_{3}+r_{1} m_{4}\right) e^{i \omega t}
$$

where $\tau \mathrm{w}$ is the wall shear stress

The couple stress coefficient at the plate is given by

$C_{w}^{\prime}=\frac{M_{w} v^{2}}{\gamma w_{0}^{3}}=P^{\prime}(0)=-r_{1} B e^{i \omega t}$

where $M_{w}$ is the plate Couple stress

The rate of heat transfer at the surface in terms of the Nusselt number is given by

$$
N u=\frac{x\left(\frac{\partial \bar{T}}{\partial \bar{z}}\right)_{\bar{z}=0}}{\left(\overline{\mathrm{T}}_{\infty}-\overline{\mathrm{T}}_{w}\right)}
$$

and on simplication Nusselt number is given by

$$
N u \operatorname{Re}_{x}^{-1}=-\theta^{\prime}(0)=r_{2} e^{i \omega t}
$$

The rate of mass transfer at the surface in terms of the Sherwood number is given by

$$
S h=\frac{x\left(\frac{\partial \bar{C}}{\partial \bar{z}}\right)_{\bar{z}=0}}{\left(\bar{C}_{\infty}-\bar{C}_{w}\right)}
$$

and on simplication Sherwood number is given by

$$
\operatorname{ShRe}_{x}^{-1}=-\phi^{\prime}(0)=\left(r_{3} a_{2}+r_{2} a_{1}\right) e^{i \omega t}
$$

\section{Results And Discussions}

The formulation of the effect of chemical reaction and Soret effect on MHD free convection heat and mass transfer flow of an incompressible micropolar fluid along a infinite vertical porous plate in a porous medium in presence of heat generation/ absorption and thermal radiation with Hall current has been performed in the preceding sections. This enables us to bring out the numerical computations for distribution of the 
translational velocity, microrotation, temperature and concentration across the boundary layer for different values of the parameters. In the current study we have chosen $M=1, m=0.3, a=0.2, G r=3, G m=1, K r=1, L=1$, $h=0.2, \beta=0.5, \operatorname{Pr}=3, Q=0.5, R=1, S c=0.2, S r=1, C r=1, n=0.5, \omega t=P i / 20$.

To verify the validity and accuracy of the present analysis, our results have been compared to the local Nusselt number and Sherwood number with those obtained by [26] for various values of .The results of this comparison are given in Table 1.It can seen from this table that excellent agreement between the results exists. This concerned comparison leads poise in the next section.

The effects of magnetic field parameter on velocity distribution profiles across the boundary layer are presented in Fig. 2. It is obvious that the effect of increasing values of the magnetic field parameter $M$ results in a decreasing velocity distribution across the boundary layer. This is due to the fact that the effect of a transverse magnetic field give rise to a resistive type force called the Lorentz force. The force has the tendency to slow the motion of the fluid. Fig.3 displays the effect of Hall current parameter (m) on the translational velocity distribution profiles. It is noticed that the Hall current parameter increases the velocity along the boundary layer. Fig. 4 shows the influence of the viscoelastic parameter on translational velocity profiles. The velocity decreases as viscoelastic parameter increases. Fig. 5 depicts the effects of permeability of the porous medium parameter $(\mathrm{Kr})$ on velocity distribution profiles and it is obvious that as permeability parameter $(\mathrm{Kr})$ increases, the velocity increases along the boundary layer thickness which is expected since when the holes of porous medium become larger, the resistivity of the medium may be neglected. Fig. 6 illustrates the variation of slip parameter with translational velocity distribution profiles.As the parameter increases the translational velocity across the boundary layer increases. It is expected since the slip parameter has the tendency to reduce the friction forces which increases fluid velocity. Fig. 7 shows the translational velocity profiles with different values of radiation parameter. And the effect of increasing the radiation parameter is to increase the translational velocity. This is because when the intensity of heat generated through thermal radiation increased, the bond holding the components of the fluid particles is easily broken and the fluid velocity will increased. The influence of the heat generation parameter $\mathrm{Q}$ on the translational velocity distribution across the boundary layer is shown in Figs. 8(a) and 8(b). Fig. 8(a) shows the translational velocity increases for smaller values of Q, but the effect is fluctuating nature near the plate and then approach to the boundary layer conditions for higher values of $Q$ as shown in Fig.8 (b). Fig.9 depicts the Soret effect ( $\mathrm{Sr}$ ) on the translational velocity distribution profiles. It is noticed that the Soret number increases the velocity profile also increases along the boundary layer. The effects of chemical reaction parameter on velocity distribution profiles across the boundary layer are presented in Fig. 10. It is obvious that the effect of increasing values of the chemical reaction parameter $\mathrm{Cr}$ results in a decreasing velocity distribution across the boundary layer.

Fig. 11 depicts the micro-rotational velocity profiles for different values of magnetic field parameter (M) respectively. The micro-rotational velocity distribution profiles decreases with increase in the magnetic field parameter. Fig. 12 illustrates the micro-rotational velocity distribution for different values of Hall current parameter $(\mathrm{m})$. The figure shows that as Hall current parameter increases, micro-rotational velocity decreases. Fig. 13 illustrates the micro-rotational velocity distribution for different values of viscoelastic parameter (a). The figure shows that as viscoelastic parameter increases, the micro-rotational velocity decreases. The effect due to permeability parameter $(\mathrm{Kr})$ on micro-rotational velocity is shown in Fig. 14. It is observed that as the parameter increases, the micro-rotational velocity decreases in the vicinity of the plate and opposite nature happens far away from the plate. Fig. 15 shows that the effect of increasing slip parameter is to increase the micro-rotational velocity in the vicinity of the plate and decrease it far away from the plate. Fig. 16 illustrates the effect of radiation parameter on micro rotational velocity profiles. The profile increases as the parameter increases. It is also noted that from Fig. 17 that the micro rotation distribution increases as Q increases. The Soret effect ( $\mathrm{Sr})$ on micro-rotational velocity is shown in Fig. 18. It is observed that as the parameter increases, the micro-rotational velocity decreases in the vicinity of the plate and reverse happens far away from the plate. Fig. 19 illustrates the micro-rotational velocity distribution for different values of chemical reaction parameter $(\mathrm{Cr})$. The figure shows that as chemical reaction parameter increases, micro-rotational velocity decreases.

Fig. 20 presents the effect of the Prandtl number Pr on the temperature profiles. Increasing the value of Pr has the tendency to decrease the fluid temperature in the boundary layer as well as the thermal boundary layer thickness. Fig. 21 illustrates the effect of radiation parameter on temperature profiles. The profile increases as the radiation parameter increases. The temperature increases with an increase of heat source parameter because when heat is generated the buoyancy forces increase the temperature profiles as shown in Fig. 22. From Fig. 23, it is reveals that concentration distribution profiles for different values of Sc. It can be noted from the figure that the concentration of the fluid decreases as the Sc increases. Fig. 24 shows the variation of concentration profiles for different values of Sr. It is observed from this figure the concentration profiles increase with increasing of Sr. Fig. 25 shows concentration distribution profiles for different values of $\mathrm{Cr}$. It can be noted from the figure that the concentration of the fluid decreases as the Sc increases. 
The numerical result for skin friction coefficient, couple stress coefficient, Nusselt number and Sherwood number are shown in Tables 2 - 4 below:

From Table 2 It is assessment that increase in value of microgyration vector to shear stress (n) decreases skin friction coefficient while couple stress coefficient increases with increase in $\mathrm{n}$. It is noticed from Table 2 that the effect of increasing values of Hall current parameter $(\mathrm{m})$, permeability parameter (Kr), radiation parameter $(\mathrm{R})$, and Soret number $(\mathrm{Sr})$ increases both skin friction coefficient and couple stress coefficient whereas reverse effect is found on increasing the values of magnetic parameter $(\mathrm{M})$, slip parameter (h), chemical reaction parameter $(\mathrm{Cr})$. Increase in heat source parameter $(\mathrm{Q})$ increases skin friction coefficient and decreases couple stress coefficient where as reverse effect is found on increasing viscoelastic parameter (a).

Table 3 shows the effect of Prandtl number ( $P r)$, radiation parameter $(\mathrm{R})$ and heat source parameter $(\mathrm{Q})$ on the Nusselt number. From Table 3 It is observed that a substantial growth in Nusselt number with increasing values of Prandtl number while reverse happen for increasing values of radiation parameter and heat source parameter.

From Table 4, it is analyzed that Sherwood number increases with an increase in Radiation parameter $(\mathrm{R})$, Heat generation parameter $(\mathrm{Q})$, Schmidt number $(\mathrm{Sc})$, and chemical reaction parameter $(\mathrm{Cr})$ and decreases with increasing value of Soret number.

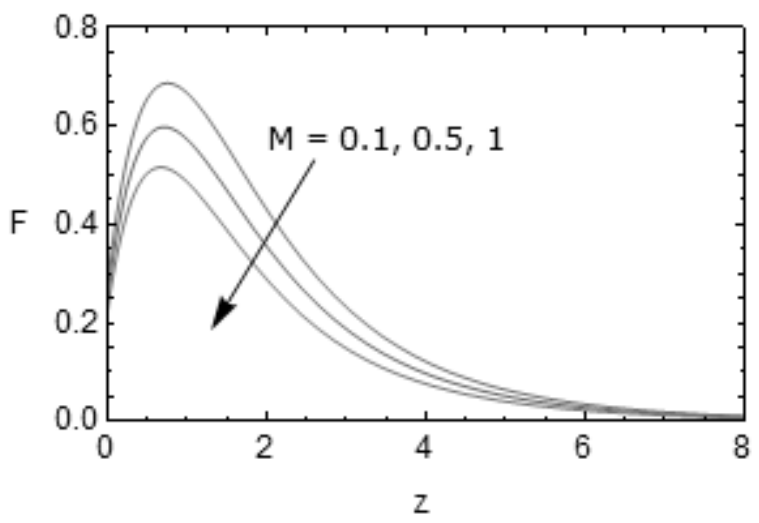

Fig 2: Velocity profile for different values of M

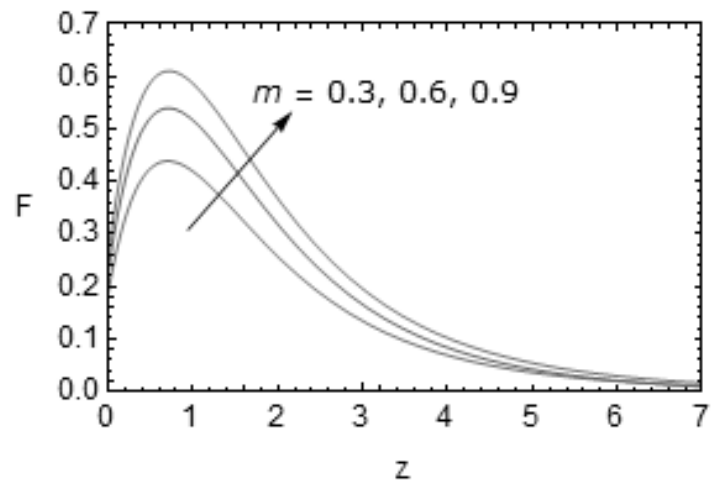

Fig 3: Velocity profile for different values of $m$

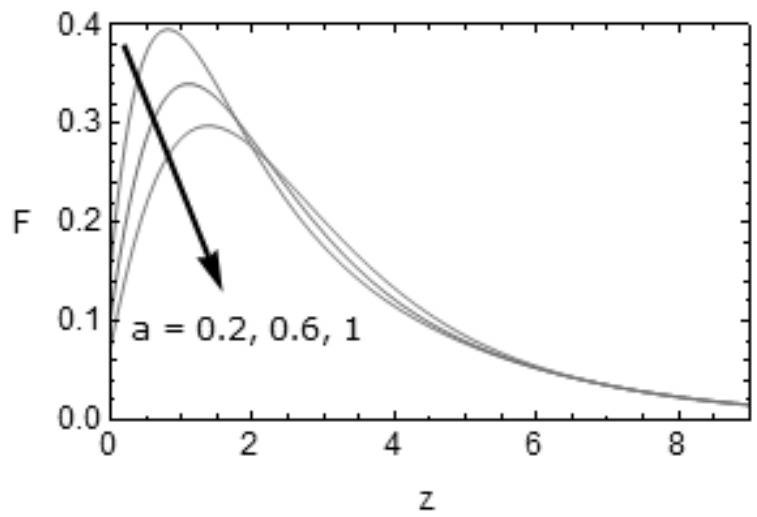

Fig 4: Velocity Profile for different values of a 


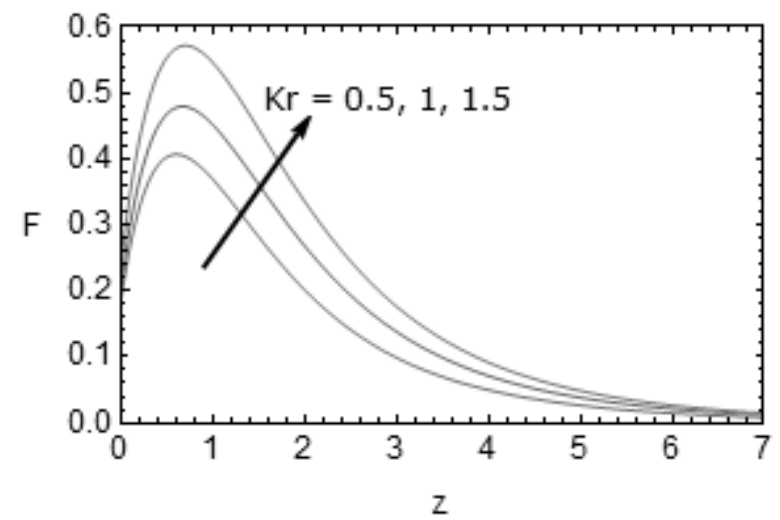

Fig 5: Velocity Profile for different values of $\mathrm{Kr}$

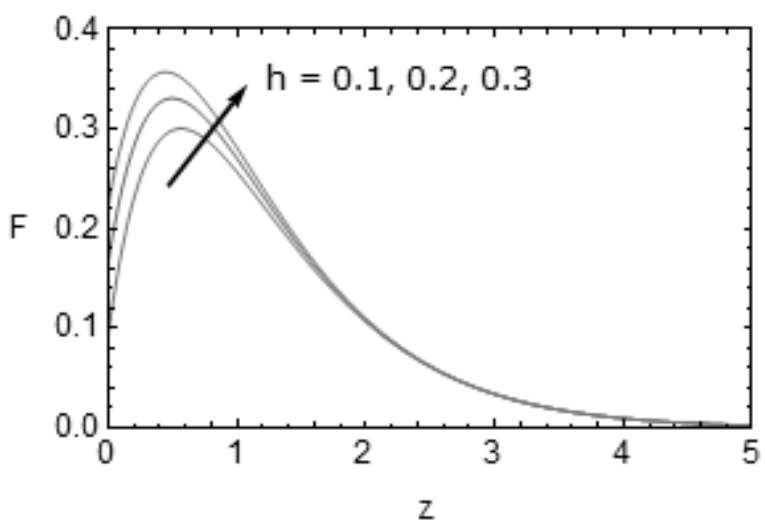

Fig 6: Velocity profile for different values of $h$

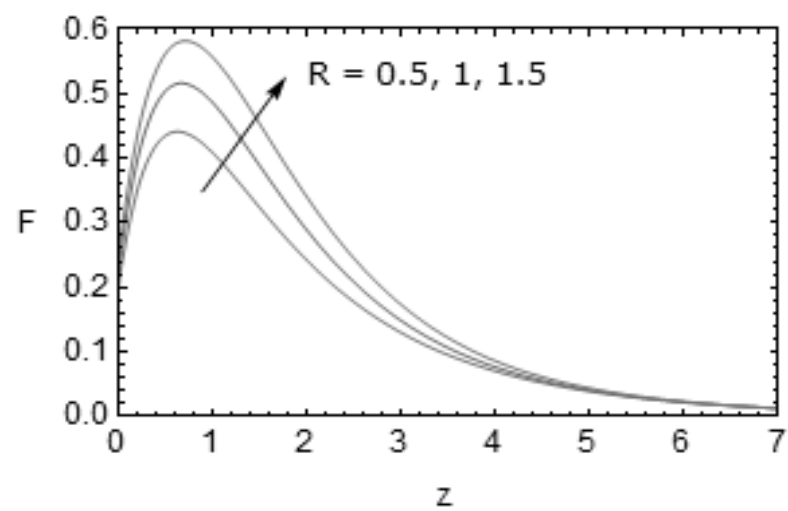

Fig 7: Velocity profile for different values $\mathrm{R}$

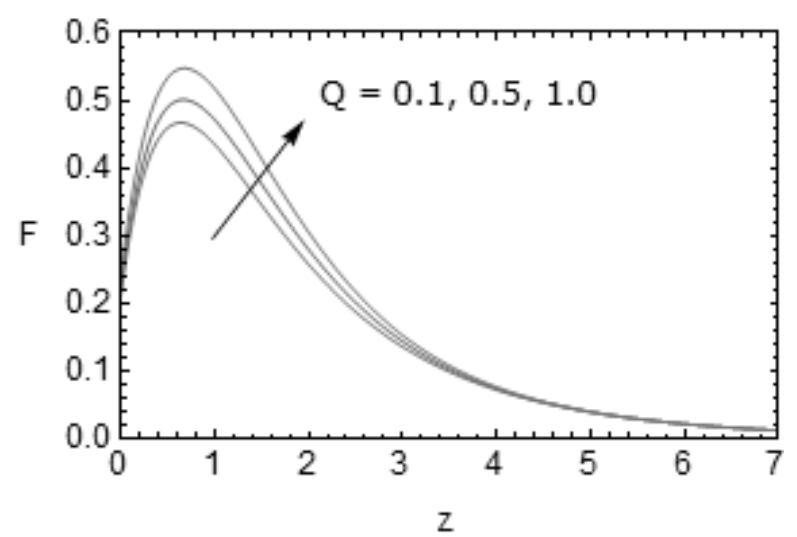

Fig.8 (a): Velocity Profile for different values of Q 


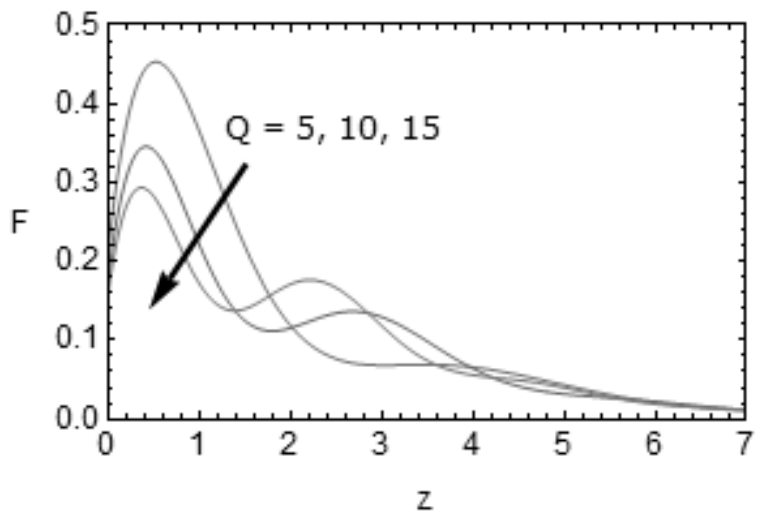

Fig 8(b): Velocity Profile for different values of Q

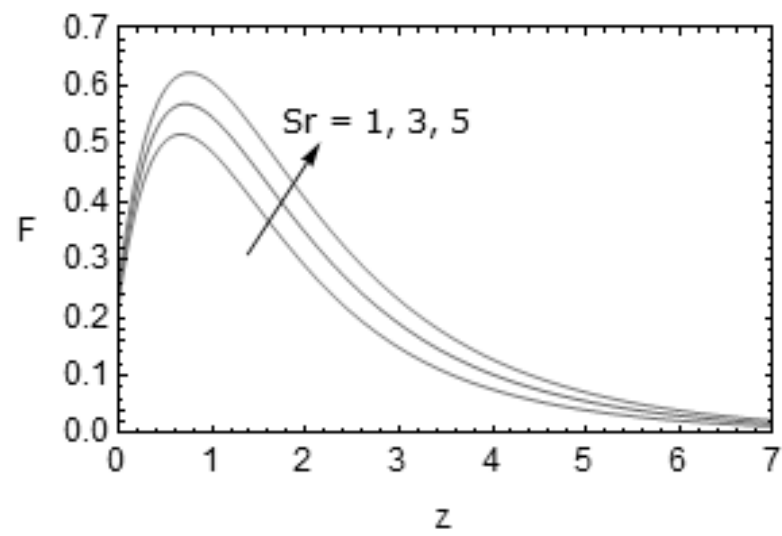

Fig.9: Velocity profile for different values of $\mathrm{Sr}$

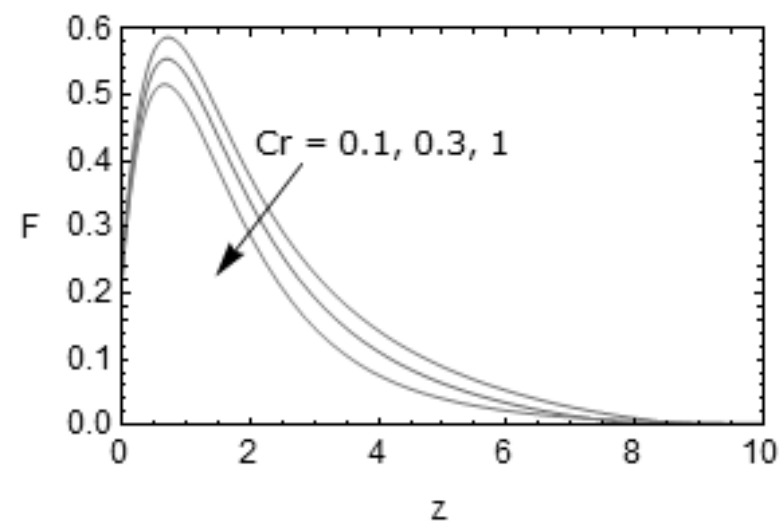

Fig.10: Velocity profile for different values of $\mathrm{Cr}$.

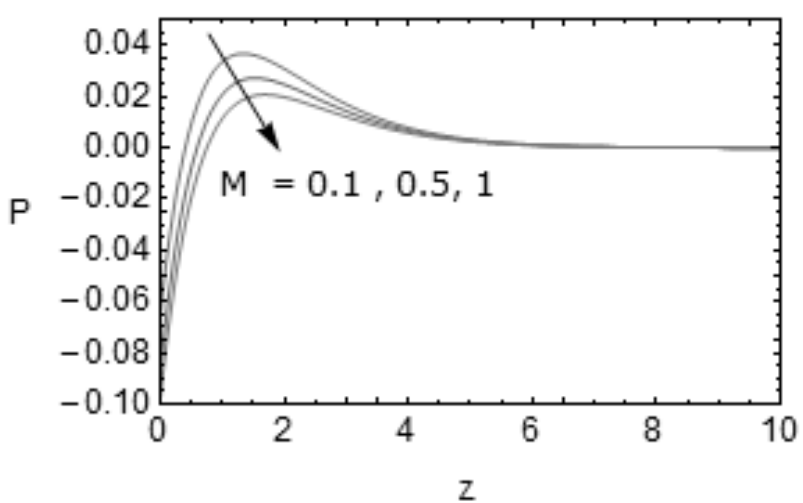

Fig.(11) :Microrotation profile for different values of M 


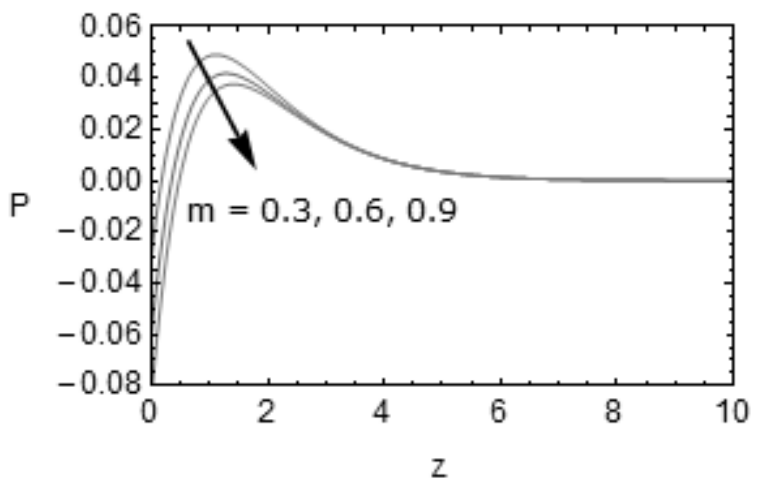

Fig.(12): Microrotation profile for different values of $m$

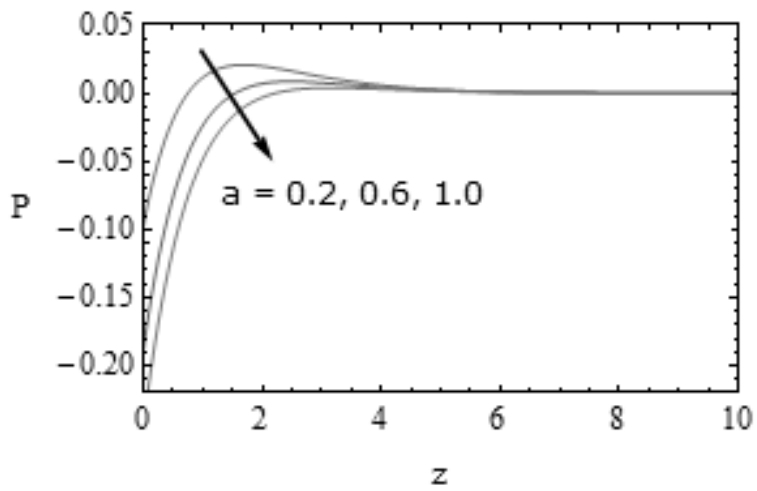

Fig.(13): Microrotation profile for different values of a

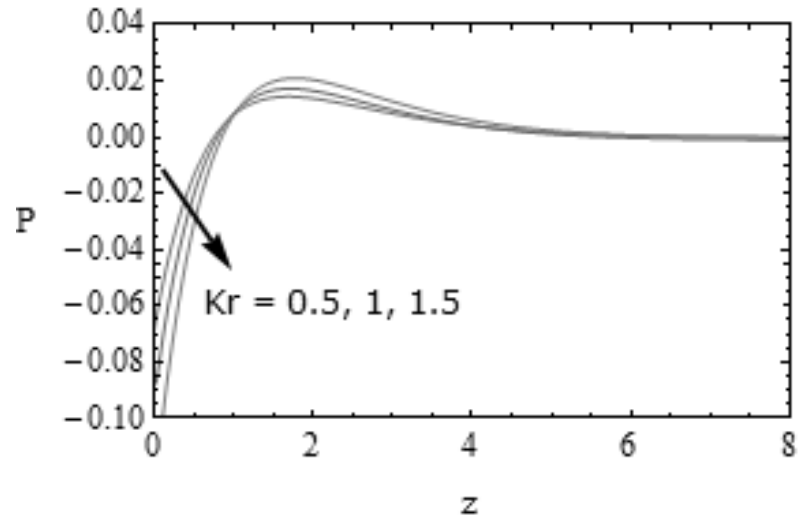

Fig.(14):Microrotation profile for different values of $\mathrm{Kr}$.

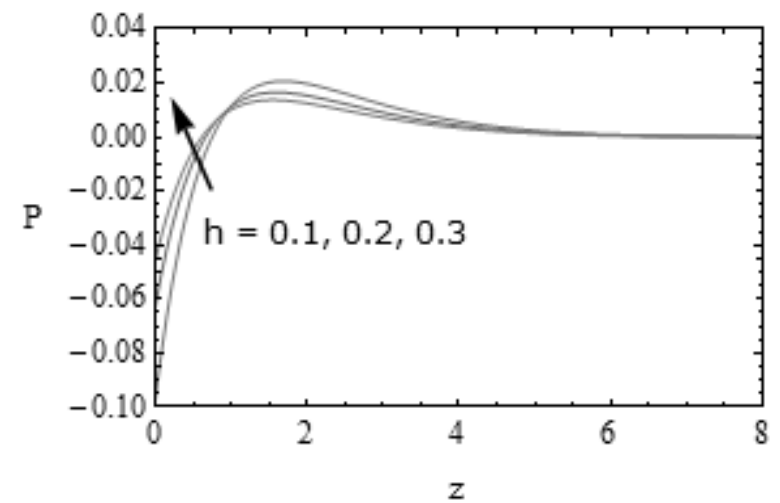

Fig. (15): Microrotation profile for different values of $h$ 


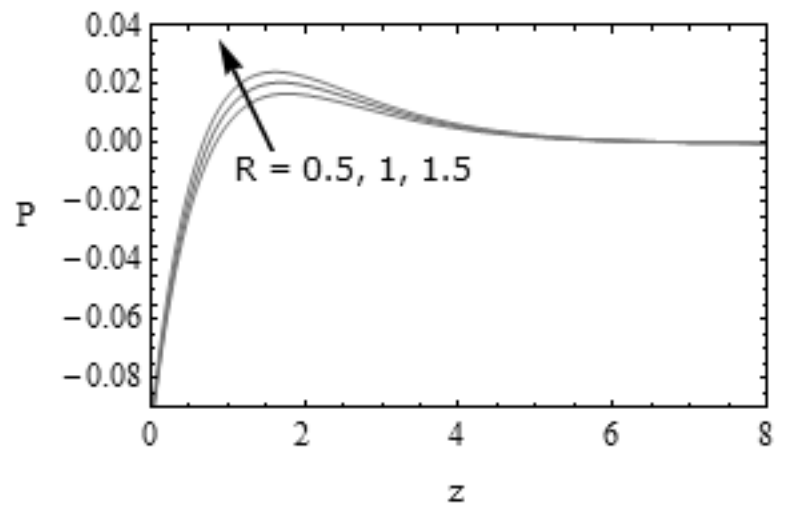

Fig.(16):Microrotation profile for different values of $R$

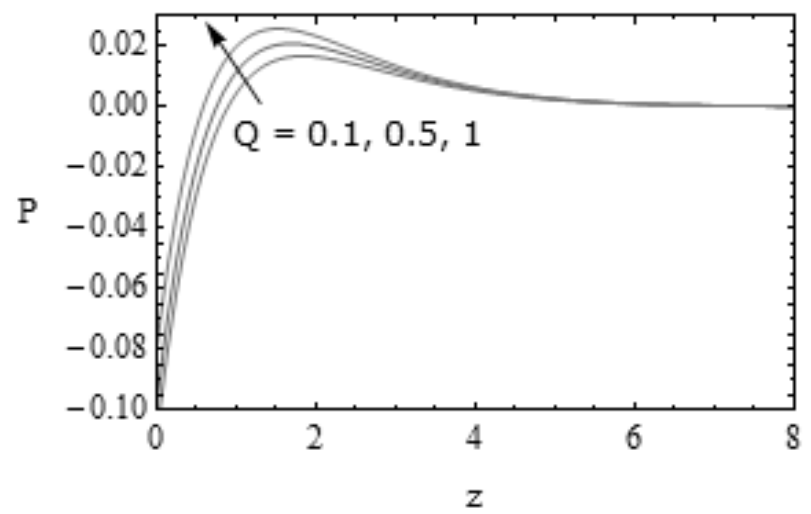

Fig.(17): Microrotation profile for different values of Q

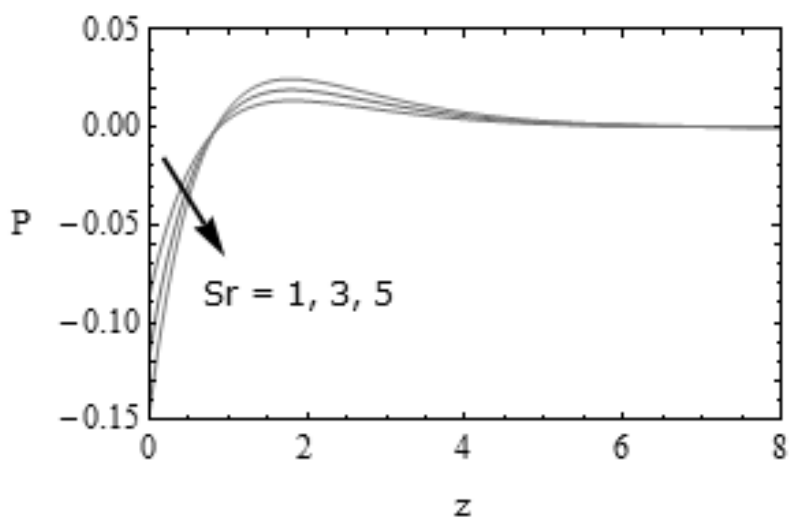

Fig.(18):Microrotation profile for different values of $\mathrm{Sr}$

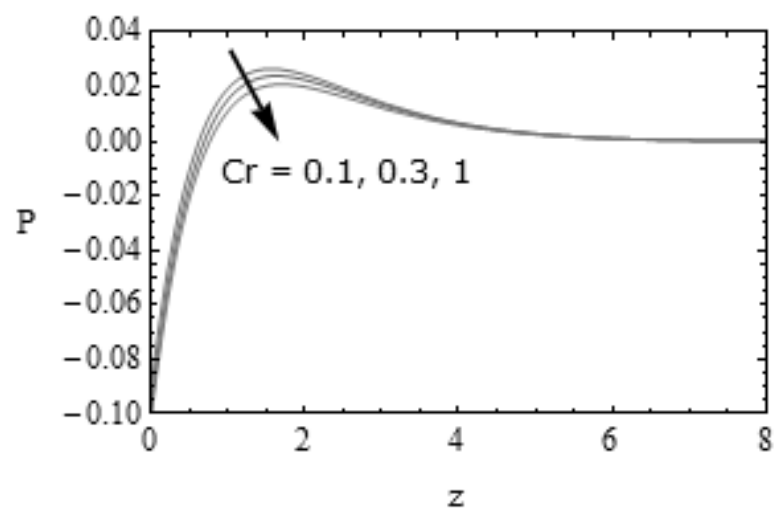

Fig.(19):Microrotation profile for different values of $\mathrm{Cr}$ 


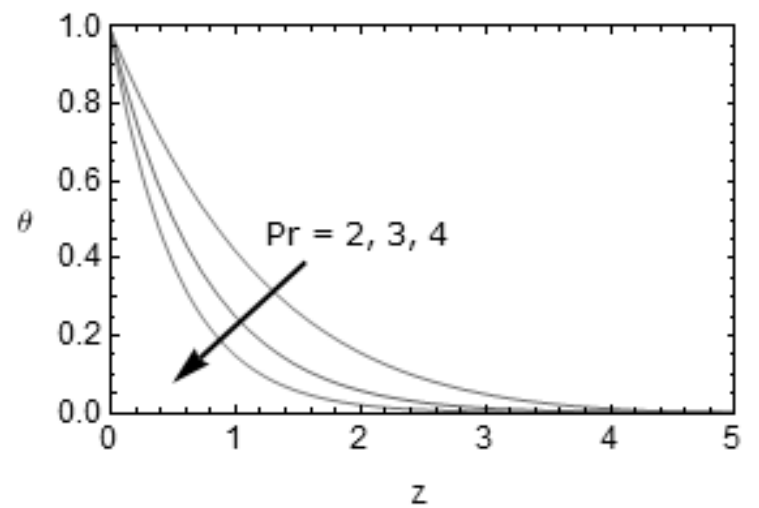

Fig. (20): Temperature profile for different values of $\mathrm{Pr}$

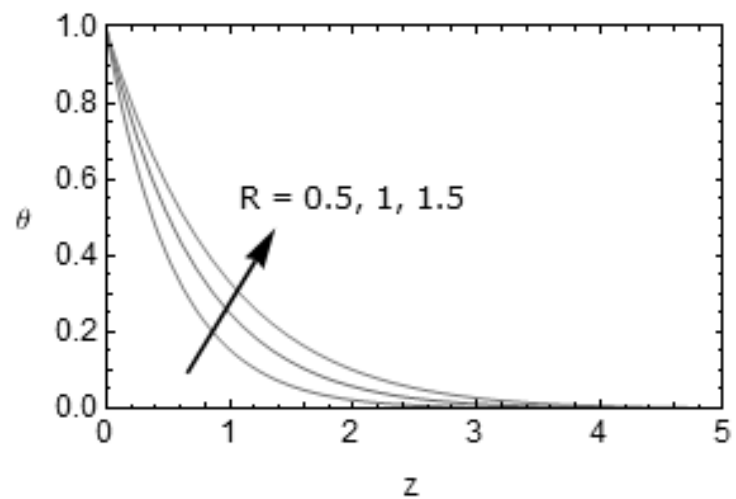

Fig.(21):Temperature profile for different values of $\mathrm{R}$

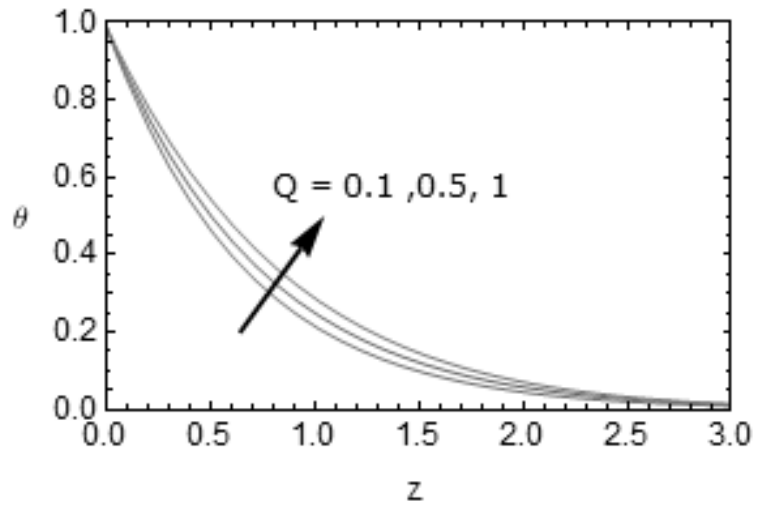

Fig.(22):Temperature profile for different values of $\mathrm{Q}$

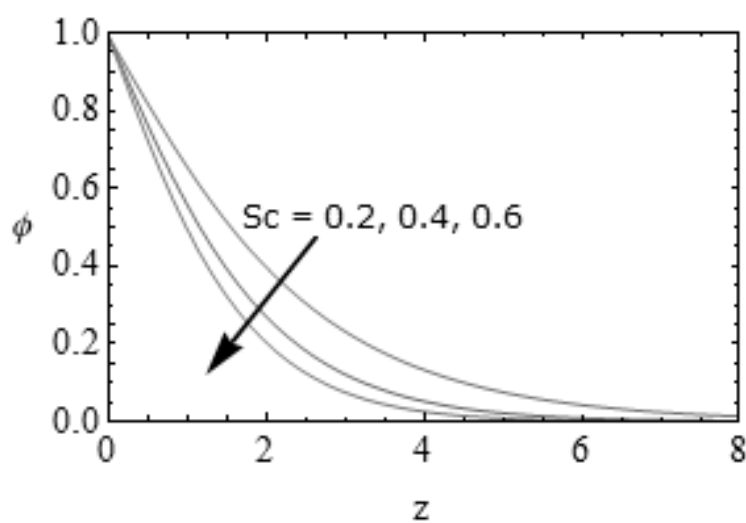

Fig.(23):Concentration profile for different values of $\mathrm{Sc}$ 


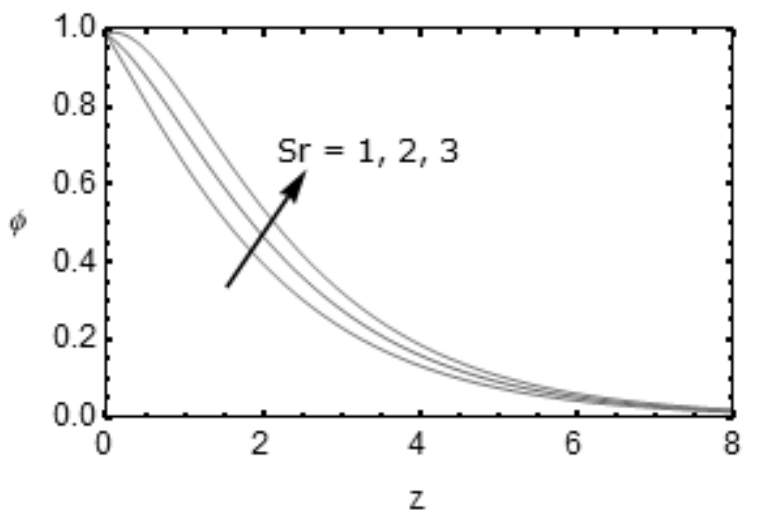

Fig.(24):Concentration profile for different values of $\mathrm{Sr}$

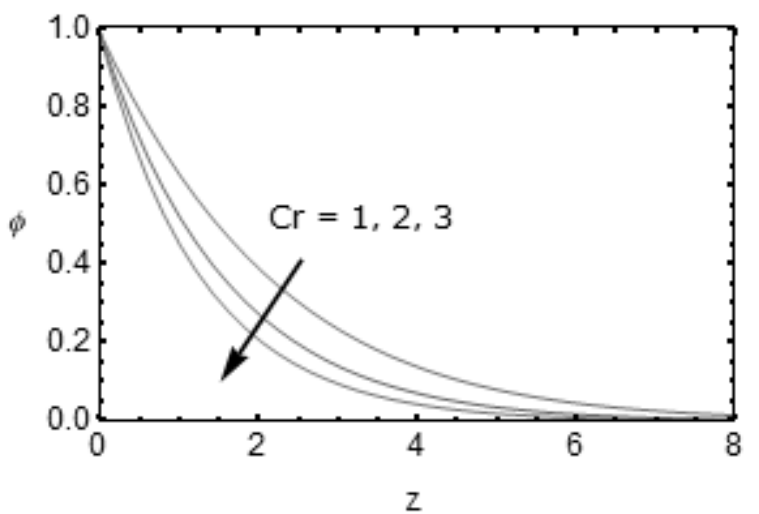

Fig.(25):Concentration profile for different values of $\mathrm{Cr}$

Table 1: Comparison of Nusselt number and Sherwood number of the present case with those of B.I.Olajuwon et al. [26] for different values of $\operatorname{Pr}, R$ and $S c$ at $\omega t=P i / 20$

\begin{tabular}{|l|l|l|l|l|l|l|}
\hline \multirow{2}{*}{ Pr } & \multirow{2}{*}{$\mathrm{R}$} & \multicolumn{2}{|c|}{ Bc } & \multicolumn{2}{c|}{ B.I.Olajuwon et al. [26] } & \multicolumn{2}{c|}{ Present Results } \\
\cline { 6 - 7 } & & & $N u R e_{x}^{-1}$ & $S h R e_{x}^{-1}$ & $N u R e_{x}^{-1}$ & $S h e_{x}^{-1}$ \\
\hline $\mathbf{3}$ & 0.5 & & 1.9661 & & 1.9661 & \\
\hline $\mathbf{4}$ & 0.5 & & 2.6176 & & 2.6176 & \\
\hline $\mathbf{5}$ & 0.5 & & 3.2716 & & 3.2716 & \\
\hline 3 & $\mathbf{0 . 2}$ & & 2.4544 & & 2.4544 & \\
\hline 3 & $\mathbf{0 . 4}$ & & 2.1054 & & 2.1054 & \\
\hline 3 & $\mathbf{0 . 8}$ & & 1.6421 & & 1.6421 & \\
\hline 3 & 0.5 & $\mathbf{2}$ & & 1.9661 & & 1.9661 \\
\hline 3 & 0.5 & $\mathbf{3}$ & & 2.9444 & & 2.9444 \\
\hline 3 & 0.5 & $\mathbf{4}$ & & 3.9271 & & 3.9271 \\
\hline
\end{tabular}

Table 2: Effect of n, M, m, a, Kr, h, R, Q, Sr, Cr on $\mathrm{Cf}$ and $\mathrm{C}_{\mathrm{w}}^{\prime}$ with $\beta=0.5, \mathrm{Gr}=3, \mathrm{Gm}=1, \mathrm{~L}=1, \mathrm{Pr}=3$,

\begin{tabular}{|l|l|l|l|l|l|l|l|l|l|l|l|}
\hline $\mathbf{n}$ & $\mathbf{M}$ & $\mathbf{m}$ & $\mathbf{a}$ & $\mathbf{K r}$ & $\mathbf{h}$ & $\mathbf{R}$ & $\mathbf{Q}$ & $\mathbf{S r}$ & $\mathbf{C r}$ & $\mathbf{C f}$ & $\mathbf{C}_{\mathbf{w}}^{\prime}$ \\
\hline $\mathbf{0 . 0}$ & 1.0 & 0.3 & 0.2 & 1.0 & 0.2 & 1.0 & 0.5 & 1.0 & 1.0 & $\mathbf{1 . 6 7 3 3}$ & $\mathbf{0 . 0 0 0 0}$ \\
\hline $\mathbf{0 . 5}$ & 1.0 & 0.3 & 0.2 & 1.0 & 0.2 & 1.0 & 0.5 & 1.0 & 1.0 & $\mathbf{1 . 3 3 8 6}$ & $\mathbf{0 . 1 8 6 4}$ \\
\hline $\mathbf{1 . 0}$ & 1.0 & 0.3 & 0.2 & 1.0 & 0.2 & 1.0 & 0.5 & 1.0 & 1.0 & $\mathbf{0 . 9 4 6 2}$ & $\mathbf{0 . 4 0 5 1}$ \\
\hline 0.5 & $\mathbf{0 . 5}$ & 0.3 & 0.2 & 1.0 & 0.2 & 1.0 & 0.5 & 1.0 & 1.0 & $\mathbf{1 . 5 0 4 7}$ & $\mathbf{0 . 1 8 9 5}$ \\
\hline 0.5 & $\mathbf{1 . 0}$ & 0.3 & 0.2 & 1.0 & 0.2 & 1.0 & 0.5 & 1.0 & 1.0 & $\mathbf{1 . 3 3 8 6}$ & $\mathbf{0 . 1 8 6 4}$ \\
\hline 0.5 & $\mathbf{1 . 5}$ & 0.3 & 0.2 & 1.0 & 0.2 & 1.0 & 0.5 & 1.0 & 1.0 & $\mathbf{1 . 2 1 2 9}$ & $\mathbf{0 . 1 8 0 0}$ \\
\hline 0.5 & 1.0 & $\mathbf{0 . 3}$ & 0.2 & 1.0 & 0.2 & 1.0 & 0.5 & 1.0 & 1.0 & $\mathbf{1 . 3 3 8 7}$ & $\mathbf{0 . 1 8 6 4}$ \\
\hline 0.5 & 1.0 & $\mathbf{0 . 6}$ & 0.2 & 1.0 & 0.2 & 1.0 & 0.5 & 1.0 & 1.0 & $\mathbf{1 . 3 8 2 4}$ & $\mathbf{0 . 2 1 2 4}$ \\
\hline 0.5 & 1.0 & $\mathbf{0 . 9}$ & 0.2 & 1.0 & 0.2 & 1.0 & 0.5 & 1.0 & 1.0 & $\mathbf{1 . 4 3 8 2}$ & $\mathbf{0 . 2 3 0 5}$ \\
\hline
\end{tabular}




\begin{tabular}{|l|l|l|l|l|l|l|l|l|l|l|l|}
\hline 0.5 & 1.0 & 0.3 & $\mathbf{0 . 2}$ & 1.0 & 0.2 & 1.0 & 0.5 & 1.0 & 1.0 & $\mathbf{1 . 3 3 8 6}$ & $\mathbf{0 . 1 8 6 4}$ \\
\hline 0.5 & 1.0 & 0.3 & $\mathbf{0 . 6}$ & 1.0 & 0.2 & 1.0 & 0.5 & 1.0 & 1.0 & $\mathbf{1 . 2 1 5 2}$ & $\mathbf{0 . 2 5 1 1}$ \\
\hline 0.5 & 1.0 & 0.3 & $\mathbf{1 . 0}$ & 1.0 & 0.2 & 1.0 & 0.5 & 1.0 & 1.0 & $\mathbf{1 . 0 4 8 0}$ & $\mathbf{0 . 2 8 4 6}$ \\
\hline 0.5 & 1.0 & 0.3 & 0.2 & $\mathbf{0 . 5}$ & 0.2 & 1.0 & 0.5 & 1.0 & 1.0 & $\mathbf{1 . 1 1 1 3}$ & $\mathbf{0 . 1 5 4 5}$ \\
\hline 0.5 & 1.0 & 0.3 & 0.2 & $\mathbf{1 . 0}$ & 0.2 & 1.0 & 0.5 & 1.0 & 1.0 & $\mathbf{1 . 3 3 8 7}$ & $\mathbf{0 . 1 8 6 4}$ \\
\hline 0.5 & 1.0 & 0.3 & 0.2 & $\mathbf{1 . 5}$ & 0.2 & 1.0 & 0.5 & 1.0 & 1.0 & $\mathbf{1 . 4 4 8 7}$ & $\mathbf{0 . 2 0 1 8}$ \\
\hline 0.5 & 1.0 & 0.3 & 0.2 & 1.0 & $\mathbf{0 . 1}$ & 1.0 & 0.5 & 1.0 & 1.0 & $\mathbf{1 . 5 2 3 7}$ & $\mathbf{0 . 2 1 7 8}$ \\
\hline 0.5 & 1.0 & 0.3 & 0.2 & 1.0 & $\mathbf{0 . 2}$ & 1.0 & 0.5 & 1.0 & 1.0 & $\mathbf{1 . 3 3 8 6}$ & $\mathbf{0 . 1 8 6 4}$ \\
\hline 0.5 & 1.0 & 0.3 & 0.2 & 1.0 & $\mathbf{0 . 3}$ & 1.0 & 0.5 & 1.0 & 1.0 & $\mathbf{1 . 1 9 3 4}$ & $\mathbf{0 . 1 6 2 6}$ \\
\hline 0.5 & 1.0 & 0.3 & 0.2 & 1.0 & 0.2 & $\mathbf{0 . 5}$ & 0.5 & 1.0 & 1.0 & $\mathbf{1 . 2 1 9 8}$ & $\mathbf{0 . 1 7 7 3}$ \\
\hline 0.5 & 1.0 & 0.3 & 0.2 & 1.0 & 0.2 & $\mathbf{1 . 0}$ & 0.5 & 1.0 & 1.0 & $\mathbf{1 . 3 3 8 6}$ & $\mathbf{0 . 1 8 6 4}$ \\
\hline 0.5 & 1.0 & 0.3 & 0.2 & 1.0 & 0.2 & $\mathbf{1 . 5}$ & 0.5 & 1.0 & 1.0 & $\mathbf{1 . 4 3 4 9}$ & $\mathbf{0 . 1 9 2 0}$ \\
\hline 0.5 & 1.0 & 0.3 & 0.2 & 1.0 & 0.2 & 1.0 & $\mathbf{0 . 1}$ & 1.0 & 1.0 & $\mathbf{1 . 3 0 3 0}$ & $\mathbf{0 . 1 8 8 3}$ \\
\hline 0.5 & 1.0 & 0.3 & 0.2 & 1.0 & 0.2 & 1.0 & $\mathbf{0 . 5}$ & 1.0 & 1.0 & $\mathbf{1 . 3 3 8 6}$ & $\mathbf{0 . 1 8 6 4}$ \\
\hline 0.5 & 1.0 & 0.3 & 0.2 & 1.0 & 0.2 & 1.0 & $\mathbf{1 . 0}$ & 1.0 & 1.0 & $\mathbf{1 . 3 8 7 9}$ & $\mathbf{0 . 1 7 7 4}$ \\
\hline 0.5 & 1.0 & 0.3 & 0.2 & 1.0 & 0.2 & 1.0 & 0.5 & $\mathbf{1 . 0}$ & 1.0 & $\mathbf{1 . 3 3 8 6}$ & $\mathbf{0 . 1 8 6 4}$ \\
\hline 0.5 & 1.0 & 0.3 & 0.2 & 1.0 & 0.2 & 1.0 & 0.5 & $\mathbf{3 . 0}$ & 1.0 & $\mathbf{1 . 4 4 9 3}$ & $\mathbf{0 . 2 0 6 6}$ \\
\hline 0.5 & 1.0 & 0.3 & 0.2 & 1.0 & 0.2 & 1.0 & 0.5 & $\mathbf{5 . 0}$ & 1.0 & $\mathbf{1 . 5 6 0 0}$ & $\mathbf{0 . 2 2 6 9}$ \\
\hline 0.5 & 1.0 & 0.3 & 0.2 & 1.0 & 0.2 & 1.0 & 0.5 & 1.0 & $\mathbf{1 . 0}$ & $\mathbf{1 . 3 3 8 6}$ & $\mathbf{0 . 1 8 6 4}$ \\
\hline 0.5 & 1.0 & 0.3 & 0.2 & 1.0 & 0.2 & 1.0 & 0.5 & 1.0 & $\mathbf{2 . 0}$ & $\mathbf{1 . 2 6 1 6}$ & $\mathbf{0 . 1 7 7 9}$ \\
\hline 0.5 & 1.0 & 0.3 & 0.2 & 1.0 & 0.2 & 1.0 & 0.5 & 1.0 & $\mathbf{3 . 0}$ & $\mathbf{1 . 2 1 3 8}$ & $\mathbf{0 . 1 7 1 6}$ \\
\hline
\end{tabular}

Table 3: Effect of Pr, R and $\mathrm{Q}$ on $N u R e_{x}^{-1}$ with $\omega \mathrm{t}=\pi / 20$

\begin{tabular}{|l|l|l|l|}
\hline Pr & R & Q & $N u R e_{x}^{-1}$ \\
\hline $\mathbf{3 . 0}$ & 1.0 & 0.5 & $\mathbf{1 . 3 1 9 3}$ \\
\hline $\mathbf{4 . 0}$ & 1.0 & 0.5 & $\mathbf{1 . 8 4 2 1}$ \\
\hline $\mathbf{5 . 0}$ & 1.0 & 0.5 & $\mathbf{2 . 3 5 4 6}$ \\
\hline 3.0 & $\mathbf{0 . 5}$ & 0.5 & $\mathbf{1 . 7 9 7 8}$ \\
\hline 3.0 & $\mathbf{1 . 0}$ & 0.5 & $\mathbf{1 . 3 1 9 3}$ \\
\hline 3.0 & $\mathbf{1 . 5}$ & 0.5 & $\mathbf{1 . 0 3 9 5}$ \\
\hline 3.0 & 1.0 & $\mathbf{0 . 1}$ & $\mathbf{1 . 4 5 0 2}$ \\
\hline 3.0 & 1.0 & $\mathbf{0 . 5}$ & $\mathbf{1 . 3 1 9 3}$ \\
\hline 3.0 & 1.0 & $\mathbf{1 . 0}$ & $\mathbf{1 . 1 4 3 1}$ \\
\hline
\end{tabular}

Table 4: Effect of R, Q, Sc, $\mathrm{Sr}$, and $\mathrm{Cr}$ on $S h R e_{x}^{-1}$ with $\mathrm{Pr}=3, \mathrm{R}=1, \mathrm{Q}=0.5$ and $\omega \mathrm{t}=\pi / 20$

\begin{tabular}{|l|l|l|l|}
\hline Sc & Sr & Cr & $S h \operatorname{Re}_{x}^{-1}$ \\
\hline $\mathbf{0 . 2}$ & 1.0 & 1.0 & $\mathbf{0 . 3 3 7 4}$ \\
\hline $\mathbf{0 . 4}$ & 1.0 & 1.0 & $\mathbf{0 . 4 5 1 7}$ \\
\hline $\mathbf{0 . 6}$ & 1.0 & 1.0 & $\mathbf{0 . 5 3 8 2}$ \\
\hline 0.2 & $\mathbf{0 . 5}$ & 1.0 & $\mathbf{0 . 4 4 1 7}$ \\
\hline 0.2 & $\mathbf{1 . 0}$ & 1.0 & $\mathbf{0 . 3 3 7 3}$ \\
\hline 0.2 & $\mathbf{1 . 5}$ & 1.0 & $\mathbf{0 . 2 3 3 0}$ \\
\hline 0.2 & 1.0 & $\mathbf{1 . 0}$ & $\mathbf{0 . 3 3 7 3}$ \\
\hline 0.2 & 1.0 & $\mathbf{2 . 0}$ & $\mathbf{0 . 5 3 8 2}$ \\
\hline 0.2 & 1.0 & $\mathbf{3 . 0}$ & $\mathbf{0 . 6 9 1 2}$ \\
\hline
\end{tabular}

\section{Conclusions}

In this endeavor, an analytical approach is used to discover the attributes of heat and mass transfer effects of an unsteady MHD flow of a chemically reacting micropolar fluid movement in existence of thermal radiation, viscoelastic, Hall current and Soret effects. The important verdicts of this enquiry are precise below:

- Thermal radiation, heat source parameter tends to enhance fluid temperature but the effect is reverse for Prandtl number while these physical quantities show opposite trend on Nusselt number. 
- The concentration profiles diminish at all points in the flow field with enhance in Schmidt number, chemical reaction parameter but are enhanced with an increase in Soret number while these physical quantities show reverse effect on Sherwood number.

- Microrotation profiles increase with an increase in radiation parameter, heat source parameter but these physical quantities show opposite nature on Couple stress coefficient.

- Slip parameter tends to enhance the Microrotation profiles in the vicinity of the plate and decreases it far away from the plate but reverse effect is found an increasing of Soret number.

- The viscoelasticity of the micropolar fluid has reducing the microrotation profiles but the reverse effect is found on couple stress coefficient.

- Microrotation profiles and couple stress coefficient decrease with an increase of chemical reaction parameter.

- Thermal radiation, heat source, and Soret number tend to enhance both translational velocity profiles and skin-friction coefficient.

- Slip parameter increases the translational velocity profiles but decreases the skin-friction coefficient.

- The translational velocity profiles and skin-friction coefficient decreases with an increase of viscoelastic parameter and chemical reaction parameter.

\section{References}

[1] A.C.Eringen, Theory of micropolar fluid, Journal of Mathematics and Mechanics, 16, 1966, 1-18

[2] A.C.Eringen, Theory of thermomicrofluids, Journal of Mathematical Analysis and Applications, 38(2), 1972, 480-496.

[3] Ariman T, Turk MA, Sylvester ND, Microcontinuum fluid mechanics-a review. Int J Eng Sci, 11, 1973, 905-930.

[4] Prathap Kumar J, Umavathi JC, Chamkha Ali J, Pop I. Fully developed free convective flow of micropolar and viscous fluids in a vertical channel. Appl Math Model, 34, 2010, 1175-86.

[5] Srinivasacharya D, Ramana murthy JV, Venugopalam D, Unsteady stokes flow of micropolar fluid between two parallel porous plates, Int J Eng Sci, 39, 2001, 1557-63.

[6] Muthuraj R, Srinivas S, Fully developed MHD flow of a micropolar and viscous fluids in a vertical porous space using HAM, Int J Appl Math Mech,6(11), 2010, 55-78.

[7] H.S.Takhar and V.M.Soundalgekar, Heat transfer in a flat plate flow of a micropolar fluid, Rheological Acta, 19, 1980, 525-526.

[8] H. S. Takhar and V. M. Soundalgekar, Flow and heat transfer of micropolar fluid past a porous plate, Indian Journal of Pure and Applied Mathematics, 16, pp. 552-558, 1985.

[9] R.S.R.Gorla, A.Slaouti, and H.S.Takhar, Free convection in micropolar fluids over a uniformly heated vertical plate, International Journal of Numerical Methods for Heat and Fluid Flow, 8(6), 1998, 504-518.

[10] R.S.R.Gorla, A.Mohammedan, M.Mansour, and I.Hussein, Unsteady natural convection from a heated vertical plate in micropolar fluid, Numerical Heat Transfer A, 28, 1995, 253-262.

[11] A.Raptis and H.S.Takhar, Polar fluid through a porous medium, Acta Mechanica, 135(1), 1999, 91-93.

[12] Y. J. Kim, Unsteady convection flow of micropolar fluids past a vertical porous plate embedded in a porous medium, Acta Mechanica, 148, 2001, 105-116.

[13] Damesh RA, Odat MQ AL, Chamka AJ, Benbella Shannk A, Combined effect of heat generation/absorption and first order chemical reaction on micropolar fluid flows over a uniform stretched permeable surface, Int J Therm Sci ,48, 2009,1658-63.

[14] Rahman MM, Al-Lawatia M, Effect of higher order chemical reaction on micropolar fluid flow on a power law permeable stretched sheet with variable concentration in a porous medium, Can J Chem Eng, 88, 2010, 23-32.

[15] Rahman MM, Aziz A, Al-Lawatia M, Heat transfer in micropolar fluid along an inclined permeable plate with variable fluid properties, Int J Therm Sci, 49, 2010, 993-1002.

[16] Sivaraj R, Rushi Kumar B, Chemically reacting dusty viscoelastic fluid flow in an irregular channel with convective boundary, Ain Shams Eng J, 4, 2012, 93-101.

[17] Abo-eldohad EM, Ghonaim AF, Radiation effect on heat transfer of a micro-polar fluid through a porous medium, Appl Math Comput 169(1), 2005, 500-16.

[18] Rahman MM, Sultana T, Radiative heat transfer flow of micropolar fluid with variable heat flux in a porous medium, Nonlinear Anal Model Control, 13(1), 2008, 71-87.

[19] Mahmoud MAA, Thermal radiation effects on MHD flow of a micropolar fluid over a stretching surface with variable thermal conductivity. Physica A, 375, 2007, 401-10.

[20] Chamkha AJ, Mohamed RA, Ahmed SE, Unsteady MHD natural convection from a heated vertical porous plate in a micropolar fluid with Joule heating, chemical reaction and radiation effects, Meccanica ,46, 2011, 399-411.

[21] N.T. Eldabe, M.E. Ouat, Chebyshew finite difference method for heat and mass transfer in hydromagnetic flow of a micropolar fluid past a stretching surface with Ohmic heating and viscous dissipation, Appl. Math. Comput. 177, 2006, 561-571.

[22] A.D. Rehbi, A.A. Tariq, A.S. Benbella, A.A. Mahoud, Unsteady natural convection heat transfer of micropolar fluid over a vertical surface with constant heat flux, Turkish J. Eng. Environ. Sci., 31, 2007, 225-233.

[23] R.C. Chaudhary, K.J. Abhay, Effect of chemical reaction on MHD micropolar fluid flow past a vertical plate in slip-flow regime, Appl. Math. Mech. Engl. Ed., 29 (9), 2008, 117-1194.

[24] K. Rakesh, C. Khem, Effect of slip conditions and Hall current on unsteady MHD flow of a viscoelastic fluid past an infinite vertical porous plate through porous medium, Int. J. Eng. Sci. Technol. 3 (4) , 2011, 0975-5462.

[25] P.V.Satya Narayana, B.Venkateswarlu, S.Venkataramana, Effects of Hall current and radiation absorption on MHD micropolar fluid in a rotating system, Ain Shams Engineering Journal, 4, 2013, 843-854

[26] B.I.Olajuwon, J.I.Oahimire, M.Ferdow, Effect of thermal radiation and Hall current on heat and mass transfer of unsteady MHD flow of a viscoelastic micropolar fluid through a porous medium, Engineering Science and Technology. 17(4), 2014, $185-193$. 